\section{CLINICAL RELEVANCE OF ADDITION OF CONVENTIONAL TREATMENT TO CONCURRENT CHEMORADIOTHERAPY IN PATIENTS WITH FIGO STAGE III-IV CERVICAL CANCER}

H Kurosu*, Y Todo, Y Suzuki, R Yamada, K Minowa, S Minobe, H Kato. National Hospital Organization, Hokkaido Cancer Center, Division of Gynecologic Oncology, Sapporo, Japan

\subsection{6/ijgc-2021-ESGO.6}

Introduction/Background* Concurrent chemoradiotherapy (CCRT) has limited therapeutic efficacy for stage III-IV cervical cancer. We aimed to identify a subgroup of patients with stage III-IV cervical cancer who benefit from CCRT with additional treatment.

Methodology We retrospectively reviewed 120 patients with stage III-IV cervical cancer who were treated with CCRT from 2002 to 2018. We compared overall survival between patients treated with CCRT alone and those who received CCRT with additional conventional treatments (systemic chemotherapy before and/or after CCRT and/or extended-field radiation). Prognostic factors were statistically analyzed.

Result(s)* Overall, 44 (36.7\%) and 21 (17.5\%) patients were radiologically diagnosed with pelvic and para-aortic lymph node enlargement, respectively. The median tumor diameter was $5.7 \mathrm{~cm}$. Sixty-nine $(57.5 \%)$ patients received no additional treatment, and $51(42.5 \%)$ received additional treatment. Cox regression analysis identified the following prognostic factors: histological non-squamous cell carcinoma (hazard ratio [HR], $3.9 ; 95 \%$ confidence interval [CI], 1.8-8.2), tumor diameter of $\geq 6 \mathrm{~cm} \quad(\mathrm{HR}, 2.1$; 95\% CI, 1.2-3.7), radiological pelvic lymph node enlargement (HR, 2.1; 95\% CI, 1.1-4.0), and radiological para-aortic lymph node enlargement (HR, 2.1; 95\% CI, 1.1-4.1). Even in the lowest risk group (no risk factors), the 5-year overall survival rate was lower in the additional treatment group than in the CCRT alone group $(78.7 \%$ vs. $80.9 \%$, respectively; log-rank test, $P=0.79$ ).

Conclusion* Addition of conventional treatments to CCRT might not improve survival in patients with advanced cervical cancer. Novel treatment strategies including immune checkpoint inhibitors should be considered for such patients.

\section{LOWER-LIMB LYMPHEDEMA AFTER SENTINEL LYMPH NODE BIOPSY IN CERVICAL CANCER PATIENTS}

${ }^{1} \mathrm{R}$ Poncová*, ${ }^{1} \mathrm{R}$ Kocián, ${ }^{2} \mathrm{~S}$ Marnitz, ${ }^{3} \mathrm{~J}$ Jarkovský, ${ }^{4} \mathrm{~J}$ Klát, ${ }^{5} \mathrm{R}$ Pilka, ${ }^{6} \mathrm{~A}$ Torné, ${ }^{7}$ I Zapardiel, ${ }^{8} \mathrm{~A}$ Petiz, ${ }^{9} \mathrm{~L}$ Lay, ${ }^{10} \mathrm{~B}$ Sehnal, ${ }^{11} \mathrm{~J}$ Ponce, ${ }^{12} \mathrm{M}$ Felsinger, ${ }^{13} \mathrm{O}$ Arencibia-Sanchéz, ${ }^{14} \mathrm{P}$ Kaščák, ${ }^{15} \mathrm{~K}$ Zalewski, ${ }^{2} \mathrm{C}$ Köhler, ${ }^{1} \mathrm{~F}$ Frühauf, ${ }^{1} \mathrm{M}$ Borčinová, ${ }^{1} \mathrm{D}$ Cibula. ${ }^{1}$ Gynecologic Oncology Center, Department of Obstetrics and Gynecology, First Faculty of Medicine, Charles University and General University Hospital, Prague, Czech Republic; ${ }^{2}$ Department of Special Operative and Oncologic Gynaecology, Asklepios-Clinic Hamburg, Hamburg, Germany; ${ }^{3}$ Institute of Biostatistics and Analyses, Faculty of Medicine, Masaryk University, Brno, Czech Republic; ${ }^{4}$ Department of Obstetrics and Gynecology, University Hospital Ostrava, Ostrava Poruba, Czech Republic (Central and Eastern European Gynecologic Oncology Group, CEEGOG); ${ }^{5}$ Department of Obstetrics and Gynecology, Faculty of Medicine and Dentistry, Palacky University, University Hospital Olomouc, Olomouc, Czech Republic (Central and Eastern European Gynecologic Oncology Group, (EEGOG); ${ }^{6}$ Unit of Gynecological Oncology, Institute Clinic of Gynaecology, Obstetrics, and Neonatology, Hospital Clinic-Institut d'Investigacions Biomediques August Pi i Sunver (IDIBAPS), University of Barcelona, Barcelona, Spain; ${ }^{7}$ Gynecologic Oncology Unit, La Paz University Hospital, Madrid, Spain; ${ }^{8}$ Instituto Portugues de Oncologia do Porto, Portugal; ${ }^{9}$ Department of Gynaecology, Institute of Oncology Angel H Roffo University of Bueno $s$ Aires, Buenos Aires, Argentina; ${ }^{10}$ Department of Obstetrics and Gynecology, University Hospital Bulovka, First Faculty of Medicine, Charles University, Praque, Czech Republic (Central and Eastern European Gynecologic Oncology Group, (EEGOG); ${ }^{11}$ Department of Gynecology, University Hospital of Bellvitge, Biomedical Research Institute of Bellvitge, (IDIBELL), University of Barcelona, Barcelona, Spain; ${ }^{12}$ Department of Gynecology and Obstetrics, Faculty of Medicine, Masaryk University, Brno, Czech Republic (Central and Eastern European Gynecologic Oncology Group, CEEGOG); ${ }^{13}$ University Hospital of the Canary Islands, Las Palmas de Gran Canaria, Spain; ${ }^{14}$ Department of Obstetrics and Gynecology, Faculty Hospital Trencin, Trencin, Slovakia (Central and Eastern European Gynecologic Oncology Group, CEEGOG);

${ }^{15}$ Department of Gynecologic Oncology, Holycross Cancer Center, Kielce, Poland (Central and Eastern European Gynecologic Oncology Group, CEEGOG)

\subsection{6/ijgc-2021-ESG0.7}

Introduction/Background* Lower-limb lymphedema (LLL) is a well-recognized adverse outcome of the surgical management of cervical cancer. Recently, sentinel lymph node (SLN) biopsy has emerged as an alternative procedure to systematic pelvic lymphadenectomy (PLND) aiming to decrease the risk of complications, especially LLL development. Our study represents the first prospective analysis of LLL incidence in cervical cancer patients after a uterine procedure with SLN biopsy, without systematic PLND.

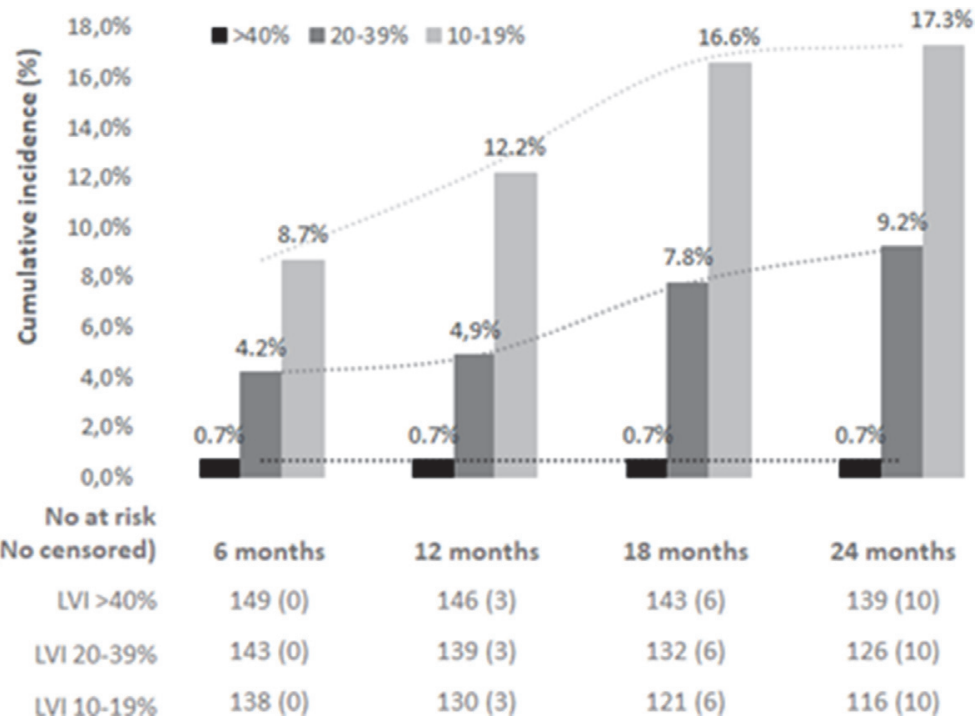




\begin{tabular}{lcc} 
& & $N(\%)$ \\
\hline Age category (years) & $\leq 40$ & $54(36.0 \%)$ \\
& $41-60$ & $78(52.0 \%)$ \\
\hline Body mass index category $\left(\mathrm{kg} / \mathrm{mg}^{2}\right)$ & $>60$ & $18(12.0 \%)$ \\
\hline FIGO stage (preoperative) & $\leq 25$ & $79(52.7 \%)$ \\
& $26-30$ & $41(27.3 \%)$ \\
& $>30$ & $30(20.0 \%)$ \\
\hline Tumor type & IA1 + LVSI & $10(6.7 \%)$ \\
& IA2 & $12(8.0 \%)$ \\
& IB1 & $128(85.3 \%)$ \\
\hline Tumor size (preoperative imaging) & Squamous cell carcinoma & $102(68.0 \%)$ \\
& Adenocarcinoma & $46(30.7 \%)$ \\
\hline Lymphovascular space invasion (LVSI) & Adenosquamous carcinoma & $2(1.3 \%)$ \\
\hline Surgical approach & $\leq 2$ cm & $107(71.3 \%)$ \\
& $>2$ cm & $43(28.7 \%)$ \\
\hline Type of uterine procedure & Yes & $40(26.7 \%)$ \\
& No & $110(73.3 \%)$ \\
\hline No of removed SLNs & Laparotomy & $50(33.3 \%)$ \\
& Minimally invasive & $100(66.7 \%)$ \\
\hline SLN metastatic involvement & Type B radical hysterectomy & $36(24.0 \%)$ \\
& Type C1 radical hysterectomy & $61(40.7 \%)$ \\
& Type C2 radical hysterectomy & $24(16.0 \%)$ \\
& Simple hysterectomy & $1(0.7 \%)$ \\
& FST (conization, trachelectomy) & $28(18.6 \%)$ \\
\hline Adjuvant treatment & 2 & $59(39.3 \%)$ \\
& $3-4$ & $69(46.0 \%)$ \\
& $>4$ & $22(14.9 \%)$ \\
\hline & No & $137(91.3 \%)$ \\
& $9(6.0 \%)$ \\
& Macrometastasis or micrometastasis & $4(2.7 \%)$ \\
\hline & Isolated tumor cells & $12(8.0 \%)$ \\
& Chemoradiotherapy & $5(3.3 \%)$ \\
& Combined radiotherapy & $1(0.7 \%)$ \\
& Brachytherapy & $132(88 \%)$ \\
\hline & None &
\end{tabular}

\section{Abstract 114 Figure 2}

Methodology In a prospective international multicenter trial SENTIX, the group of 150 patients with stage IA1-IB2 cervical cancer treated by uterine surgery with bilateral SLN biopsy was prospectively evaluated using both objective LLL assessments, based on limb volume increase (LVI) between pre- and postoperative measurements, and subjective patient-perceived swelling were conducted in six-month periods over 24-months post-surgery. The characteristics of the patients are summarized in table 1.

Result(s)* The cumulative incidence of LLL at 24 months was $17.3 \%$ for mild LLL (LVI 10-19\%), 9.2\% for moderate LLL (LVI 20-39\%), while only one patient (0.7\%) developed severe LLL (LVI >40\%). The median interval to LLL onset was nine months (figure 1). A transient edema resolving without intervention within six months was reported in an additional $22 \%$ of patients. Subjective LLL was reported by $10.7 \%$ of patients, though only a weak and partial correlation between subjectivereport and objective-LVI was found. No risk factor directly related to LLL development was identified.

Conclusion* Contrary to the expectations, the replacement of standard PLND by bilateral SLN biopsy in the surgical treatment of cervical cancer does not eliminate the risk of mild to moderate LLL, which develops irrespective of the number of SLN removed.

Trial registration ClinicalTrials.gov: NCT02494063

Funding This work was supported by Charles University in Prague (UNCE 204065 and PROGRES Q28/LF1) and by a grant from the Czech Health Research Council (NV19-0300023). The funders had no role in the design of the study; in the collection, analyses, or interpretation of data; in the writing of the manuscript, or in the decision to publish the results.

Conflicts of Interest The authors declare no conflict of interest.

\section{EVALUATION OF BINTRAFUSP ALFA, A BIFUNCTIONAL FUSION PROTEIN TARGETING TGF- $\beta$ AND PD-L1, IN CERVICAL CANCER: DATA FROM PHASE 1 AND PHASE 2 STUDIES}

1J Strauss ${ }^{*},{ }^{2} \mathrm{~F}$ Braiteh, ${ }^{3} \mathrm{E}$ Calvo Aller, ${ }^{3} \mathrm{M}$ De Miguel, ${ }^{4} \mathrm{~A}$ Cervantes, ${ }^{5} \mathrm{WJ}$ Edenfield, ${ }^{6} \mathrm{~T} \mathrm{LI}$, ${ }^{7} \mathrm{MA}$ Rasschaert, ${ }^{8} \mathrm{TW}$ Park-Simon, ${ }^{9} \mathrm{FL}$ Munoz, ${ }^{10} \mathrm{~L}$ Paz-Ares, ${ }^{11} \mathrm{~A}$ Spira, ${ }^{12} \mathrm{G}$ Jehl, ${ }^{13}$ I Dussault, ${ }^{13} \mathrm{~L}$ Ojalvo, ${ }^{14} \mathrm{~J}$ Gulley, ${ }^{15} \mathrm{~S}$ Allan. ${ }^{1}$ Center for Cancer Research, National Cancer Institute, National Institutes of Health, Laboratory of Tumor Immunology and Biology, Bethesda, MD, USA; ${ }^{2}$ Comprehensive Cancer Centers of Nevada, Las Vegas, NV, USA; ${ }^{3}$ START Madrid-CIOCC, Centro Integral Oncológico Clara Campal, Madrid, Spain; ${ }^{4}$ University of Valencia, Biomedical Research Institute INCLIVA, València, Spain; ${ }^{5}$ Greenville Hospital System University Medical Center (ITOR), Greenville, SC, USA; ${ }^{6}$ University of California Davis Comprehensive Cancer Center, Sacramento, CA, USA; 'Universitair Ziekenhuis Antwerpen, Antwerpen, Belgium; ${ }^{8}$ Medizinische Hochschule Hannover, Hannover, Germany; ${ }^{9}$ Hospital Universitario Ramón y Cajal, Instituto Ramón y Cajal de Investigación Sanitaria, CIBERONC, Madrid, Spain; ${ }^{10}$ Hospital Universitario 12 de Octubre, Madrid, Spain; ${ }^{11}$ Virginia Cancer Specialists, Fairfax, VA, USA; ${ }^{12}$ Merck KGaA, Darmstadt, Germany; ${ }^{13}$ EMD Serono Research and Development Institute, Inc., Billerica, MA, USA; ${ }^{14}$ Center for Cancer Research, National Cancer Institute, National Institutes of Health, Genitourinary Malignancies Branch, Bethesda, MD, USA; ${ }^{15}$ Tasman Oncology Research Ltd, Southport, QLD, Australia

\subsection{6/ijgc-2021-ESG0.8}

Introduction/Background* The accelerated US Food and Drug Administration approval of pembrolizumab validated the efficacy of anti-PD-(L)1 therapy for patients with recurrent/metastatic cervical cancer; however, the objective response rate (ORR) with pembrolizumab was $14.3 \%$ in patients with PDL1-expressing tumours. Human papillomavirus infection is implicated in $>95 \%$ of cervical cancers and is linked to

\begin{tabular}{|c|c|}
\hline & $\begin{array}{l}\text { All patients } \\
(\mathrm{N}=39)\end{array}$ \\
\hline Best overall response, $\mathrm{n}(\%)$ & $2(5.1)$ \\
\hline Complete response (CR) & $9(23.1)$ \\
\hline Partial response (PR) & $3(7.7)$ \\
\hline Stable disease & $20(51.3)$ \\
\hline Progressive disease (PD) & $5(12.8)$ \\
\hline Not evaluable & $1(2.6)$ \\
\hline \multicolumn{2}{|l|}{ Delayed PR* } \\
\hline Confirmed ORR (CR + PR), n (\%) & $11(28.2)$ \\
\hline $95 \% \mathrm{Cl}$ & $15.0-44.9$ \\
\hline Total clinical response rate (ORR + delayed PR), $\mathrm{n}(\%)$ & $12(30.8)$ \\
\hline Confirmed ORR in subgroups, $\mathrm{n} / \mathrm{n}(\%)$ & $6 / 24(25.0)$ \\
\hline Squamous cell carcinoma & $5 / 12(41.7)$ \\
\hline Adenocarcinoma & $6 / 25(24.0)$ \\
\hline Prior bevacizumab & $5 / 14(35.7)$ \\
\hline \multicolumn{2}{|l|}{ No prior bevcizumab } \\
\hline Duration of response (confirmed ORR), median (range), months & $11.7(1.4-41.2)$ \\
\hline Ongoing response, $n / n(\%)$ & $5 / 11(45.5)$ \\
\hline Duration of ongoing response, months (range) & $1.4-41.2$ \\
\hline Median overall survival, months & 13.4 \\
\hline $95 \% \mathrm{Cl}$ & 5.5-not reached \\
\hline 24-month overall survival rate, $\%$ & 33.2 \\
\hline
\end{tabular}

